\title{
DFT Investigation of the Hydrogen Adsorption on Graphene and Graphene Sheet Doped with Osmium and Tungsten
}

\author{
Balqees Suliman Alshareef \\ Faculty of Science, Chemistry Department, King Abdulaziz University, Jeddah, Saudi Arabia \\ Email: nwazzan@kau.edu.sa
}

How to cite this paper: Alshareef, B.S. (2020) DFT Investigation of the Hydrogen Adsorption on Graphene and Graphene Sheet Doped with Osmium and Tungsten. Open Journal of Physical Chemistry, 10, 197-204. https://doi.org/10.4236/ojpc.2020.104012

Received: July 20, 2020

Accepted: November 3, 2020

Published: November 6, 2020

Copyright $\odot 2020$ by author(s) and Scientific Research Publishing Inc. This work is licensed under the Creative Commons Attribution International License (CC BY 4.0).

http://creativecommons.org/licenses/by/4.0/

\section{(c) (i) Open Access}

\begin{abstract}
Significant interest has been focused on graphene materials for their unique properties as Hydrogen storage materials. The development of their abilities by modifying their configuration with doped or decorated transition metals was also of great interest. In this work, using the DFT/B3LYP/6-31G/LanL2DZ level of theory, graphene sheet (GS) as one of the materials of interest was doped with two transition metals, Osmium (Os) and Tungsten (W). Two active sites on the GS were tested (C4 and C16) resulted into adsorbed systems, H2@C4-GS and H2@C16-GS. C16 position showed the largest adsorption energy compared to that at $\mathrm{C} 4$. Therefore, $\mathrm{C} 4$ was replaced by the two metals and two adsorbed systems were formed: $\mathrm{H}_{2} @ \mathrm{O}$-GS and H2@W-GS. The binding energy of $\mathrm{H}_{2} @ \mathrm{O}-\mathrm{GS}$ was found to be greater than that of H2@W-GS.
\end{abstract}

\section{Keywords}

Graphene Sheet, Doping Metal, Osmium, Tungsten, Density Functional Theory, Adsorption, The Highest Occupied Molecular Orbital, The Lowest Unoccupied Molecular Orbital

\section{Introduction}

To make a promising solution for hydrogen storage materials, carbon materials are considered [1] [2] [3]. Graphene as one of the most important carbon materials was successfully made and demonstrated to be stable via structuring the carbon atoms by sp $^{2}$-bonding [4] [5]. Moreover, graphene sheets (GSs) attracted extensive interest from the researchers due to their low cost, lighter weight, special nano-surface structures, electrical properties, and wide industrial applications, gas storage as an example [2] [6] [7]. Hydrogen gas was more preferred 
due to its sustainability and low mass density for that the U.S. Department of Energy (DOE) has set a target value of $9 \mathrm{wt} \%$ hydrogen storage by 2015, which is far from today's possibly approaching value $6.5 \mathrm{wt} \%$ by 2020 [7] [8]. However, two main problems are found in the graphene as hydrogen gas storage that blocks the ability to apply it, and they are: 1) the low efficiency of pure graphene in the storage process, and 2) the weakness of the intermolecular interaction that affects the storage. Many studies show that doping a transition metal on the GS would significantly enhance the hydrogen storage binding energies with respect to the pure graphene sheet [1] [6].

What we are aiming to pursue in this work is to investigate another way of metal-doping graphene to improve the adsorption of the hydrogen. We nominated Osmium (Os) metal in order to be doped with graphene. Since, previous studies show that Os has the highest binding ability to GS [6], in which the Os metal was placed above the GS bonded to two carbon atoms. In this work, different configuration has been adopted, in which one of the carbon atoms was replaced by an Os atom. In addition, in another configuration, a Tungsten (W) atom was used in a similar way. For the best of the present author knowledge, the two adopted configurations with the two metals have not been reported yet. We hope that this research could add a value point and the promising information in the field. The GS doped with TMs is abbreviated as Os-GS and W-GS, while the adsorbed systems of $\mathrm{H}_{2}$ molecule on the GS and doped GS are abbreviated as $\mathrm{H}_{2} @ G S, \mathrm{H}_{2} @ \mathrm{O} s-\mathrm{GS}$, and $\mathrm{H}_{2} @ \mathrm{~W}-\mathrm{GS}$. Our results show enhancement in the adsorption energies of systems of H2@Os-GS, and H2@W-GS H2 in comparison to undoped GS ( $\left.\mathrm{H}_{2} @ \mathrm{GS}\right)$.

\section{Computational Details}

In order to obtain the optimized geometries, binding energies, and HOMO-LUMO orbitals of the adsorbed systems, the density functional theory (DFT) [1] [2] [3] [8] method was performed using Becke 3-Parameter (Exchange), Lee, Yang and Parr (B3LYP), functional with a the 6-31G(d) basis set a split-valence double zeta basis set enlarged with one polarized basis function; a d-type orbital was added to all atoms except the hydrogen atoms, and the Los Alamos National Laboratory 2-Double-Zeta (LanL2DZ) basis set for transition metals [6] [9] [10]. All calculations were carried out using Gaussian 09 [11] software package in conjunction with GaussView version 5.0 [12].

The adsorption energies $\left(\Delta E_{\mathrm{ads}}\right)$ of the hydrogen molecule adsorbed on the pure GS and TM-doped Gs can be obtained from Equations (1) and (2), respectively.

$$
\begin{gathered}
\Delta E_{\mathrm{ads}}=E_{\mathrm{H} 2 @ \mathrm{GS}}-\left(E_{G S}+E_{\mathrm{H} 2}\right) \\
\Delta E_{\mathrm{ads}}=E_{\mathrm{H} 2 @ \mathrm{TM}-\mathrm{G}}-\left(E_{\mathrm{TM}-\mathrm{G}}+E_{\mathrm{H} 2}\right)
\end{gathered}
$$

where $E_{\mathrm{H} 2 @ \mathrm{GS}}$ and $E_{\mathrm{H} 2 @ \mathrm{TM}-\mathrm{GS}}$ are total energies of the adsorption of hydrogen molecule on TM-doped to GS, respectively. $E_{\mathrm{GS}}$ and $E_{\mathrm{TM}-\mathrm{GS}}$ are total energies of the GS and TM-doped to GS, respectively, and $E_{\mathrm{H} 2}$ is the total energies of the iso- 
lated hydrogen molecule.

\section{Results and Discussion}

\subsection{Determination the Reactive Carbon Sites of GS}

Graphene sheet (GS) is a two-dimensional structure with 24-C in hexagonal pattern was chosen. To explore the most reactive carbon site, two carbon positions were chosen, $\mathrm{C} 16$ and $\mathrm{C} 4$ as shown in Figure 1. In order to mimic the adsorption of the hydrogen molecule on the GS, the $\mathrm{H}_{2}$ molecule was placed in close distance to $\mathrm{C} 4 / \mathrm{C} 16$ position. The obtained adsorption energies as calculated according to Equation (2.1) are listed in Table 1. The result shows that the adsorption energy of the hydrogen adsorbed at $\mathrm{C} 4\left(\mathrm{H}_{2} @ \mathrm{C} 4-\mathrm{GS}\right)$ is -0.0306 $\mathrm{kcal} / \mathrm{mol}$ and it is better than the hydrogen adsorbed at C16 ( $\left.\mathrm{H}_{2} @ \mathrm{C} 16-\mathrm{GS}\right)$ that have adsorption energy of $-0.0300 \mathrm{kcal} / \mathrm{mol}$. Therefore, the adsorbed system of (H2@C4-GS) is adopted to carry out the remaining calculations.

\subsection{Geometrical Parameters of Adsorbed Systems of $\mathrm{H}_{2} @ \mathrm{GS}$ and $\mathrm{H}_{2} @$ TM-GS}

In order to dope the GS with the TM, the C4 position in GS is replaced by a TM (Os/W). The optimized geometries of six systems: GS, $\mathrm{H}_{2} @ G S$, Os-GS, $\mathrm{H}_{2} @ \mathrm{Os}-\mathrm{GS}, \mathrm{W}-\mathrm{GS}$ and $\mathrm{H}_{2} @ \mathrm{~W}-\mathrm{GS}$ are calculated at the B3LYP with basis set 6-31G(d)/LanL2DZ as shown in Figure 2. The optimized parameters included the bond lengths, bond angles, hydrogen distance, and binding distance are listed in Table 1.
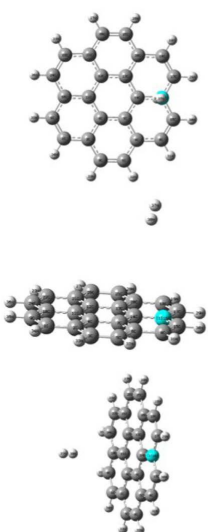

(a)
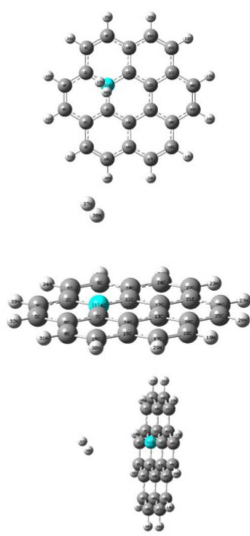

(b)

Figure 1. Hydrogen molecule attached to the graphene sheet (a) $\mathrm{H}_{2} @ \mathrm{C} 16-\mathrm{GS}$; and (b) $\mathrm{H}_{2} @ \mathrm{C} 4-\mathrm{GS}$.

Table 1. Adsorption energies (in $\mathrm{kcal} / \mathrm{mol}$ ) and their components (in Hartree) of hydrogen molecule adsorped at the two positions (C4 and C16) of graphene sheet.

\begin{tabular}{ccccc}
\hline Adsorbed system & $\mathrm{H}_{2} @ \mathrm{GS}$ & $\mathrm{H}_{2}$ & $\mathrm{GS}$ & $\Delta E_{\text {ads }}$ \\
\hline $\mathrm{H}_{2} @ \mathrm{C} 16-\mathrm{GS}$ & -923.073 & -1.175 & -921.898 & -0.0300 \\
$\mathrm{H}_{2} @ \mathrm{C} 4-\mathrm{GS}$ & -923.073 & -1.175 & -921.898 & -0.0306
\end{tabular}




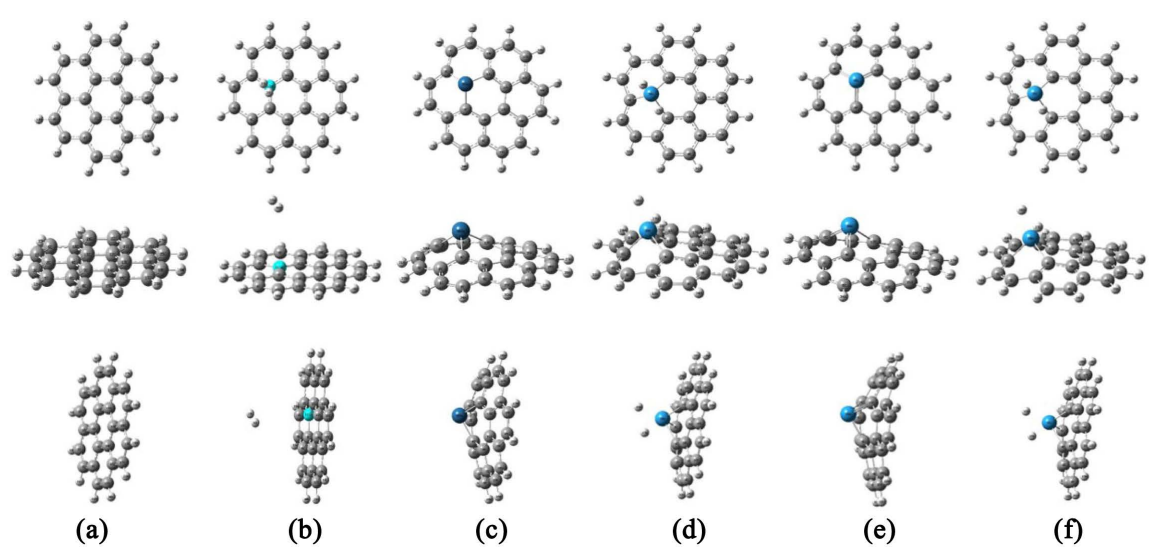

Figure 2. The optimized structures of (a) pure GS; (b) $\mathrm{H}_{2} @ G S$; (c) Os-GS; (d) $\mathrm{H}_{2} @ O$ Os-GS; (e) W-GS and (f) $\mathrm{H}_{2} @$ W-GS.

When the TM atom replaces the $\mathrm{C} 4$ position, the planarity of the molecule is distorted. Since, the TS (Os/W) appeared somehow above the plane of the other atoms of GS, due the greater atomic size of the TM atom compared to that of the carbon atom. Such position of the TS allows easier approaching and thus adsorption of the $\mathrm{H}_{2}$ species. In other words, the presence of protruding structures in the adsorption process can reduce steric hindrance effect, resulting in an increased ability of adsorption. Similar observation can be found in literature [1] [3] [6] [8].

The calculation in Table 2, produced the bond lengths of $\mathrm{H}_{2}$ at graphene sheet ( $\mathrm{H}_{2} @ \mathrm{GS}$ ) denoted by C4-C2, C7-C4, C4-C11, C7-C14 and C7-C6 that were estimated to be $1.421,1.427,1.427,1.4276$ and $1.421 \AA$, respectively. However, the bond lengths increase when transition metals added to GS. Generally, the bond lengths in Os-doped to GS are lower than those in the W-doped to GS. Moreover, the bond lengths of $\mathrm{H}_{2} @ O s-G S$ system denoted by Os-C13, Os-C6, Os-C4, C13-C15 and C4-C10 were estimated to be 1.930, 1.917, 1.946, 1.426 and 1.432 $\AA$, respectively. In addition, the bond lengths of $\mathrm{H}_{2} @ \mathrm{~W}-\mathrm{GS}$ denoted by W-C13, W-C6, W-C4, C13-C15 and C4-C10 are 1.937, 1.928, 2.122, 1.425 and $1.434 \AA$, respectively. Therefore, the bond lengths increase due to the replacement by a transition metals in compared to those in pure GS. The increase in the bond lengths follows the order: $\mathrm{H}_{2} @ W-G S>\mathrm{H}_{2} @ O$ Os-GS > $\mathrm{H}_{2} @ G S$. The increase in these lengths is due to the difference in electronegativity of Os (2.2) and W (2.36). We expect that two factors lead to the observed increase in bond lengths, i.e., the lower electronegativity and atomic size of Os in comparison to those of $\mathrm{W}[6]$.

On the other hand, the bond angels of $\mathrm{H}_{2}$ at pure $\mathrm{GS}\left(\mathrm{H}_{2} @ \mathrm{G}\right)$ are $119.994^{\circ}$, $120.005^{\circ}, 120.009^{\circ}, 120.001^{\circ}$ and $118.780^{\circ}$ for C11-C4-C7, C4-C7-C14, C2-C4-C7, C4-C11-C12 and C4-C2-C1, respectively. The bond angels of $\mathrm{H}_{2}$ at Os-GS ( $\mathrm{H}_{2} @ \mathrm{Os}-\mathrm{GS}$ ) denoted by C13-Os-C6, Os-C4-C2, C4-Os-C13, Os-C13-C15 and C4-Os-C6 were estimated to be $92.764^{\circ}, 120.146^{\circ}, 90.476^{\circ}, 118.752^{\circ}$ and $93.621^{\circ}$, respectively. While, for W-GS ( $\left.\mathrm{H}_{2} @ \mathrm{~W}-\mathrm{GS}\right)$ the bond angle for C13-W-C6, 
Table 2. Selected geometrical parameters, $\mathrm{H}-\mathrm{H}$ distance, and binding distance of $\mathrm{H}_{2}$ adsorbed on pure GS, Os-doped and W-doped at GS with $\mathrm{H}-\mathrm{H}$ distances of isolated hydrogen, calculated at B3LYP/6-31G(d)/LanL2DZ.

\begin{tabular}{|c|c|c|c|c|c|c|}
\hline \multirow[t]{2}{*}{ System } & \multicolumn{2}{|c|}{ Bond lengths/Å } & \multicolumn{2}{|c|}{ Bond angles $/^{\circ}$} & \multirow[t]{2}{*}{$\mathrm{H}-\mathrm{H}$ distance } & \multirow[t]{2}{*}{ Binding distance } \\
\hline & $\mathrm{C} 4-\mathrm{C} 2$ & 1.421 & C11-C4-C7 & 119.994 & & \\
\hline \multirow{4}{*}{$\mathrm{H}_{2} @ \mathrm{GS}$} & $\mathrm{C} 7-\mathrm{C} 4$ & 1.427 & C4-C7-C14 & 120.005 & \multirow{4}{*}{$\begin{array}{c}\mathrm{H} 35-\mathrm{H} 37 \\
0.742\end{array}$} & \multirow{4}{*}{$\begin{array}{c}\mathrm{H} 37-\mathrm{C} 4 \\
4.141\end{array}$} \\
\hline & C4-C11 & 1.427 & C2-C4-C7 & 120.009 & & \\
\hline & C7-C14 & 1.427 & C4-C11-C12 & 120.001 & & \\
\hline & C7-C6 & 1.421 & $\mathrm{C} 4-\mathrm{C} 2-\mathrm{C} 1$ & 118.780 & & \\
\hline \multirow{5}{*}{$\mathrm{H}_{2} @ \mathrm{Os}-\mathrm{GS}$} & Os-C13 & 1.930 & C13-Os-C6 & 92.764 & \multirow{5}{*}{$\begin{array}{c}\mathrm{H} 38-\mathrm{H} 37 \\
0.827\end{array}$} & \multirow{5}{*}{$\begin{array}{c}\text { H37-Os } \\
1.945\end{array}$} \\
\hline & Os-C6 & 1.917 & Os-C4-C2 & 120.146 & & \\
\hline & Os-C4 & 1.946 & C4-Os-C13 & 90.476 & & \\
\hline & C13-C15 & 1.426 & Os-C13-C15 & 118.752 & & \\
\hline & $\mathrm{C} 4-\mathrm{C} 10$ & 1.432 & C4-Os-C6 & 93.621 & & \\
\hline \multirow{5}{*}{$\mathrm{H}_{2} @ \mathrm{~W}-\mathrm{GS}$} & W-C13 & 1.937 & C13-W-C6 & 99.009 & \multirow{5}{*}{$\begin{array}{c}\mathrm{H} 36-\mathrm{H} 37 \\
2.016\end{array}$} & \multirow{5}{*}{$\begin{array}{c}\mathrm{H} 36-\mathrm{W} \\
1.781\end{array}$} \\
\hline & W-C6 & 1.928 & $\mathrm{~W}-\mathrm{C} 4-\mathrm{C} 2$ & 118.416 & & \\
\hline & $\mathrm{W}-\mathrm{C} 4$ & 2.122 & $\mathrm{C} 4-\mathrm{W}-\mathrm{C} 13$ & 81.525 & & \\
\hline & $\mathrm{C} 13-\mathrm{C} 15$ & 1.425 & W-C13-C15 & 102.114 & & \\
\hline & C4-C10 & 1.434 & C4-W-C6 & 86.539 & & \\
\hline
\end{tabular}

W-C4-C2, C4-W-C13, W-C13-C15 and C4-W-C6 were estimated to be $99.009^{\circ}$, $118.416^{\circ}, 81.525^{\circ}, 102.114^{\circ}$ and $86.539^{\circ}$, respectively. The increasing in bond angles is in the following order: $\mathrm{H}_{2} @ O$ Os-GS $<\mathrm{H}_{2} @ W-G S<\mathrm{H}_{2} @ G S$. Another calculated values are the $\mathrm{H}-\mathrm{H}$ distance, for $\mathrm{H}_{2}$ at pure $\mathrm{GS}\left(\mathrm{H}_{2} @ \mathrm{G}\right)$ denoted by H35-H37 is $0.74276 \AA$ and for $\mathrm{H}_{2}$ at OS-GS ( $\left.\mathrm{H}_{2} @ O s-G S\right)$ denoted by H38-H37 that were estimated to be $0.827 \AA$. While, $\mathrm{H}-\mathrm{H}$ distance of $\mathrm{H}_{2}$ at W-GS ( $\mathrm{H}_{2} @ W-G S$ ) denoted by H36-H37 is $2.016 \AA$. The H-H distances are elongated due to the doping process, and follow the order: $\mathrm{H}_{2} @ \mathrm{GS}<\mathrm{H}_{2} @ \mathrm{Os}-\mathrm{GS}<$ $\mathrm{H}_{2} @ \mathrm{~W}-\mathrm{GS}$.

The binding distance of $\mathrm{H}_{2}$ of $\mathrm{H}_{2} @ \mathrm{G}, \mathrm{H}_{2} @ \mathrm{O}$-GS, and $\mathrm{H}_{2} @ \mathrm{~W}-\mathrm{GS}$ denoted by H37-C4, H37-Os, and H36-W were estimated to be 4.1411, 1.9452, and 1.7814 $\AA$, respectively. Decreasing of binding distance of $\mathrm{H}_{2}$ can be seen after adding the transition metal to GS. This indeed will lead to improvement in the adsorption energies. The decrease in the binding distances follows the order: $\mathrm{H}_{2} @ \mathrm{~W}-\mathrm{GS}<$ $\mathrm{H}_{2} @ \mathrm{Os}-\mathrm{GS}<\mathrm{H}_{2} @ \mathrm{GS}$. The shorting in the binding distance is expected to lead to a strong adsorption and higher adsorption energy. Therefore, the adsorption to the doped GS is expected to be stronger compared to bare GS, as will prove later.

\subsection{Adsorption Energies of Adsorbed Systems of H2@GS and H2@TM-GS}

The adsorption energies for H2@GS and H2@TM-GS systems are calculated according to equations 1 and 2 and listed in Table 3. From there, the values of the 
adsorption energies $\left(\Delta E_{\mathrm{ads}}\right)$ were computed and they are $-0.031,-8.369$ and $-1.538 \mathrm{kcal} / \mathrm{mol}$, respectively. In addition, stability effect on the adsorption process of the TM-doped is significant. However, $\Delta E_{\text {ads }}$ of $\mathrm{H}_{2} @ \mathrm{Os}-\mathrm{G}(-8.3693$ $\mathrm{kcal} / \mathrm{mol})$ are more stable than $\mathrm{H}_{2} @ \mathrm{~W}-\mathrm{G}(-1.537 \mathrm{kcal} / \mathrm{mol})$. Therefore, the stability order of the three adsorbed systems is: $\mathrm{H}_{2} @ \mathrm{O}-\mathrm{G}>\mathrm{H}_{2} @ \mathrm{~W}-\mathrm{G}>\mathrm{H}_{2} @ \mathrm{G}$. This result showed the superiority of Os-doped to GS compared to W [6].

\subsection{Frontier Molecular Orbitals}

The highest occupied molecular orbital (HOMO) and the lowest unoccupied molecular orbital (LUMO) [6], of GS and doped GS before and after the adsorption process were visualized and presented in Figure 3. The HOMO and LUMO orbitals are delocalized over the surface of undoped GS with and without the $\mathrm{H}_{2}$ molecule. On the other hand, in the doped systems and before and after the adsorption process, the HOMO orbitals are spreading on the whole system. Also, the LUMO orbitals are spreading on the whole system but mainly delocalized on the TS and $\mathrm{H}_{2} @ \mathrm{TS}$ moieties, which indicated the easier intramolecular/intermolecular electron transfer within the doped systems.

\section{Conclusion}

The B3LYP/6-31G(d)/LanL2DZ method/basis sets calculations were performed to obtain the geometrical parameters and adsorption energies of the adsorbed systems of bare GS and doped-GS (with Os and W) with molecular hydrogen. The results show that the adsorption energies of the doped systems are larger

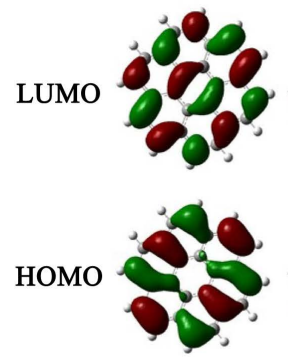

(a)
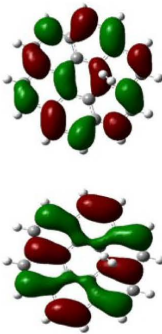

(b)
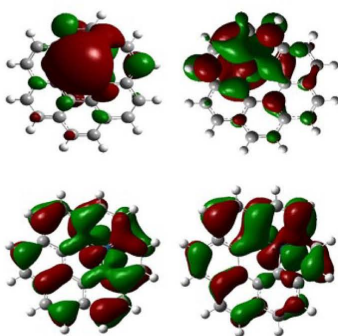

(c)
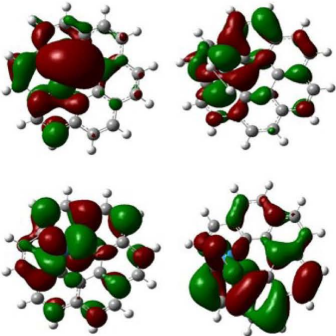

(e)

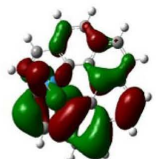

(f)

Figure 3. Visualized HOMO and LUMO orbitals of the pure GS and hydrogen molecule adsorbed on GS and Os-doped and W-doped as calculated at the B3LYP/6-31G(d)/ LanL2DZ level of theory. (a) GS; (b) $\mathrm{H}_{2} @ G S$; (c) Os-GS; (d) $\mathrm{H}_{2} @ O s-G S ;$ (e) W-GS and (f) $\mathrm{H}_{2} @ \mathrm{~W}-\mathrm{GS}$.

Table 3. Adsorption energies and their components (in $\mathrm{kcal} / \mathrm{mol}$ ) of $\mathrm{H}_{2}$ molecule adsorbed on pure GS and TM-doped.

\begin{tabular}{ccccc}
\hline adsorbed system & Gs/Os-GS/W-GS & $\mathrm{H}_{2}$ & GS/W-GS/Os-GS & $\Delta E_{\mathrm{ads}}$ \\
\hline $\mathrm{H}_{2} @ \mathrm{GS}$ & -923.073 & -1.175 & -921.898 & -0.030 \\
$\mathrm{H}_{2} @ \mathrm{Os}-\mathrm{GS}$ & -975.870 & -1.175 & -974.681 & -8.369 \\
$\mathrm{H}_{2} @ \mathrm{~W}-\mathrm{GS}$ & -952.611 & -1.175 & -951.379 & -1.537 \\
\hline
\end{tabular}


than that of the undoped system. In addition, the Os-doped system has the higher adsorption energy by $8.339 \mathrm{kcal} / \mathrm{mol}$ with respect to that of bare GS system, and by $6.832 \mathrm{kcal} / \mathrm{mol}$ with respect to that of W-GS system. Therefore, the GS doped with Os and W, specifically the Os, can be used as promising materials for hydrogen storage. Moreover, previous experimental studies performed electronic transport studies of graphene with a dilute coating of osmium adatoms, due to the potential for inducing a strong spin-orbit coupling, and the experimental results show that osmium is found to donate holes to graphene, otherwise, the graphene transport is impacted in similar fashion to other metallic anatomy, in that the scattering appears consistent with isolated coulomb impurities a small distance above the surface [13]. That could motivate us to plan for further studies on Os-GS at new position and compare it with other transition metals to explore the $\mathrm{H} 2$ adsorption possibility.

\section{Acknowledgements}

I would like to thank my supervisor Dr. Nuha Wazzan, and Ms. Ohoud Al-Qurashi for all the help and guidance they provided throughout this research.

\section{Conflicts of Interest}

The author declares no conflicts of interest regarding the publication of this paper.

\section{References}

[1] Radovic, L.R. (2005) The Mechanism of $\mathrm{CO}_{2}$ Chemisorption on Zigzag Carbon Active Sites: A Computational Chemistry Study. Carbon, 43, 907-915. https://doi.org/10.1016/j.carbon.2004.11.011

[2] Nachimuthu, S., Lai, P.-J. and Jiang, J.-C. (2014) Efficient Hydrogen Storage in Boron Doped Graphene Decorated by Transition Metals-A First-Principles Study. Carbon, 73, 132-140. https://doi.org/10.1016/j.carbon.2014.02.048

[3] Liu, W., Liu, Y. and Wang, R. (2014) Prediction of Hydrogen Storage on Y-Decorated Graphene: A Density Functional Theory Study. Applied Surface Science, 296, 204-208. https://doi.org/10.1016/j.apsusc.2014.01.087

[4] Novoselov, K.S., et al. (2004) Electric Field Effect in Atomically Thin Carbon Films. Science, 306, 666-669. https://doi.org/10.1126/science.1102896

[5] Novoselov, K.S., et al. (2005) Two-Dimensional Gas of Massless Dirac Fermions in Graphene. Nature, 438, 197-200. https://doi.org/10.1038/nature04233

[6] Tabtimsai, C., Rakrai, W. and Wanno, B. (2017) Hydrogen Adsorption on Graphene Sheets Doped with Group 8B Transition Metal: A DFT Investigation. Vacuum, 139, 101-108. https://doi.org/10.1016/j.vacuum.2017.02.013

[7] Boateng, E. and Chen, A. (2020) Recent Advances in Nanomaterial-Based Solid-State Hydrogen Storage. Materials Today Advances, 6, Article ID: 100022. https://doi.org/10.1016/j.mtadv.2019.100022

[8] Mohan, M., et al. (2019) Hydrogen Storage in Carbon Materials-A Review. Energy Storage, 1, e35. https://doi.org/10.1002/est2.35

[9] Lewars, E. (2003) Computational Chemistry. Introduction to the Theory and Ap- 
plications of Molecular and Quantum Mechanics, p. 318.

[10] Cramer, C.J. (2013) Essentials of Computational Chemistry: Theories and Models. John Wiley \& Sons, Hoboken.

[11] Gaussian09. (2020) https://gaussian.com/glossary/g09

[12] Gauss view5.0. (2020) https://gaussview.soft112.com/

[13] Elias, J.A. and Henriksen, E.A. (2020) Unexpected Hole Doping of Graphene by Osmium Adatoms. Annalen der Physik, 532, Article ID: 1900294.

https://doi.org/10.1002/andp.201900294 